\title{
Pneumatic Artificial Muscle Actuated Robot for Lower Limb Rehabilitation Triggered by Electromyography Signals Using Discrete Wavelet Transformation and Support Vector Machines
}

\author{
Chih-Jer Lin, ${ }^{*}$ Ho-Chiao Chuang, ${ }^{1}$ Chao-Wei Hsu, ${ }^{1}$ and Chin-Sheng Chen \\ Graduate Institute of Automation Technology, National Taipei University of Technology, \\ 1, Sec. 3, Zhongsiao E. Road, Taipei 10608, Taiwan, R.O.C. \\ ${ }^{1}$ Department of Mechanical Engineering, National Taipei University of Technology, \\ 1, Sec. 3, Zhongsiao E. Road, Taipei 10608, Taiwan, R.O.C.
}

(Received May 1, 2017; accepted September 29, 2017)

Keywords: pneumatic artificial muscle, electromyography, discrete wavelet transformation, support vector machines, rehabilitation

In this study, a real-time electromyography (EMG)-triggered controller for a pneumatic artificial muscle (PAM) actuated lower limb rehabilitation robot is proposed. To make the rehabilitation task controllable by the patient's movement intention, a novel trigger controller is designed according to the EMG signals of the patient's muscle. For predicting his or her movement intention in advance, the EMG signals of the patient's muscle are captured and identified to realize the proposed EMG-triggered control. To guarantee the safety and performance of the proposed system, a patient's movement intention must be identified accurately by EMG feature extraction. First, the discrete wavelet transformation (DWT) technique is used to acquire the feature vectors of the EMG signals. The properties of the different feature spaces are taken into consideration, and the optimal multicomponents of features are chosen according to the experimental results. Second, support vector machines (SVMs) are studied to improve the classification performance. Finally, the MyRIO controller is used to implement a closed-loop control system for the rehabilitation robot with the movement-intention trigger control.

\section{Introduction}

The rehabilitation of stroke patients, those with spinal cord injuries (SCIs), patients who underwent total knee replacement (TKR) surgery, the elderly, and those with other movement disorders has become a major social problem owing to the lack of physical therapists (PTs), because conventional manual therapy requires experienced therapists. However, the aging society with fewer children has resulted in manpower shortage, so that the lack of therapists makes it difficult to meet the requirements of high-intensity and repetitive rehabilitation for patients. ${ }^{(1)}$ Therefore, rehabilitation robotics has become a research field that has attracted increasing attention over the last decade. Rehabilitation robots can reduce the heavy burden of PTs and can evaluate patients' recovery status by analyzing their bioelectrical data.

\footnotetext{
*Corresponding author: e-mail: cjlin@ntut.edu.tw
} http://dx.doi.org/10.18494/SAM.2017.1736 
There are several published review papers surveying the mechanical design, control strategies, and training modes of rehabilitation robots. For example, Diaz et al. conducted a comprehensive survey about current lower limb rehabilitation robots, but they did not emphasize training modes and control strategies. ${ }^{(2)}$ Kawakkel et al. discussed control strategies for rehabilitation robots and focused on the clinical outcomes of different systems. ${ }^{(3)}$ Mohammed and coworkers reviewed the actuated exoskeletons of lower limb wearable robots and focused on the control strategies in them. ${ }^{(4,5)}$ Meng et al. presented a review and analysis of mechanisms, training modes, and control strategies of lower limb rehabilitation robots; they mainly focused on control methods with hybrid data fusion. ${ }^{(6)}$

Pneumatic artificial muscles (PAMs) were first developed by McKibben in the 1950s for use in artificial limbs; therefore, they are also called the McKibben artificial muscle. ${ }^{(7)}$ The PAM is a device like a braided pneumatic actuator with an inner rubber tube and a layer of nonexpendable braided shell. The tube is wrapped in a double-helix-braided shell woven at a predetermined angle. Both ends of the rubber tube are closed with caps. When compressed gas enters the tube through a hose connected to a pressure source, the PAM contracts in the longitudinal direction according to its inflation volume. Over the last decade, robotics engineers have begun to use them as actuators of rehabilitation robots. Costa et al. proposed a powered lower limb orthosis whose actuators are PAMs and they used highly compliant actuators (PAMs) to manipulate the exoskeleton. ${ }^{(8)}$

The design philosophy provided an important insight into the rehabilitative orthosis systems. Sawicki and Fessis in 2009 developed the pneumatically powered knee-ankle-foot orthosis (KAFO), which was proposed through the study of human motor adaptation, gait rehabilitations, and locomotion energetics. ${ }^{(9)}$ In 2011, Park et al. developed a lower-limb rehabilitation orthosis, which is made of a bio-inspired active soft orthotic device for ankle foot pathology. ${ }^{(10)}$ In 2012, Teng et al. developed a KAFO using McKibben actuators to provide assistance during gait training. ${ }^{(11)}$ In 2013, Hussain et al. inverted a robotic orthosis with six degrees of freedom for use in gait training. ${ }^{(12)}$ Arzuwan et al. in 2014 presented a survey of existing lower-limb leg orthoses for rehabilitation using pneumatic muscle-type actuators, such as McKibben artificial muscles, air muscles, PAMs or pneumatic muscle actuators (PMAs). ${ }^{(13)}$ Although many review papers had surveyed gait training, control strategies, mechanisms of rehabilitation robotics, and patients' biosignals, they did not discuss the details about how to trigger the rehabilitation robotics. Therefore, we focus on how to identify patients' movement intention using electromyography (EMG) signals and to realize the proposed EMG-triggered control in this study.

An EMG signal is generated before the patient makes a limb muscle contraction. Without feature extraction and pattern recognition, surface EMG cannot be used to identify the user's intention but provides only a limited assessment of muscle activity. The EMG signals acquired from muscles require advanced methods such as filtering, processing, decomposition, and classification. The purpose of this study is to illustrate the algorithms of feature extraction and pattern recognition for EMG signal analysis to provide effective ways of identifying the user's movement intention in advance. Then, the self-designed rehabilitation robot would operate with a predefined trajectory after being activated. The proposed PAM-actuated lower-limb rehabilitation using EMG-triggered control is implemented using the embedded real-time controller and experimental results are analyzed and discussed.

\section{Proposed EMG-Triggered Control for Lower Limb Rehabilitation Robot}

The proposed PAM actuated rehabilitation robotic system consists of two subsystems, namely, a cognition system integrated with EMG sensors and a rehabilitation robot (RRS) system as shown 
in Fig. 1. The first subsystem is the cognitive human-robot interaction (cHRi), which consists of EMG sensors, a signal processing card, a data acquisition (DAQ) card, and a PC to deal with feature extraction and classification. The second subsystem is the proposed RRS. The RRS is actuated by PAMs and controlled by the real-time embedded system (MyRIO) using the proposed controller algorithm. The details about the RRS and cHRi are described as follows.

\subsection{Design of PAM actuated rehabilitation robot}

Most lower-limb rehabilitation orthosis systems use conventional actuators, such as DC or AC brush motors, DC brushless servomotors, linear actuators, and pneumatic cylinders. However, compared with conventional pneumatic cylinders and motors, PAMs have several advantages, including compactness, inherent compliance, low cost, and high power-to-weight ratio. ${ }^{(14)}$ Moreover, PAMs are highly compliant actuators to manipulate the rehabilitation exoskeleton; thus, they are safer than other actuators such as motors. Compared with other complicated rehabilitation orthosis systems, the main purpose of our design is concentrated on knee rehabilitation. Figure 2 shows the mechanism design of the proposed rehabilitation robot, which has two degrees of freedom for gait training tasks. The knee and hip joints are both attached to pulleys as shown in

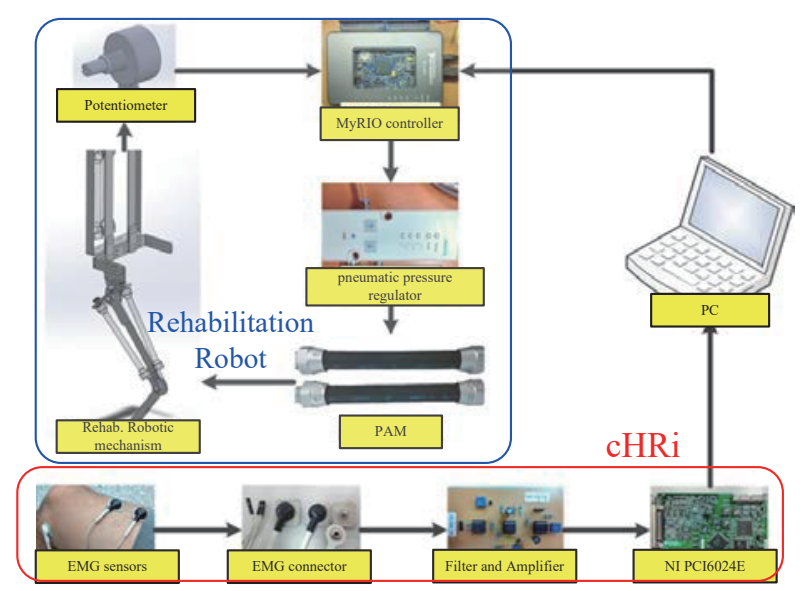

Fig. 1. (Color online) Experimental setup and systematic scheme used in this study.

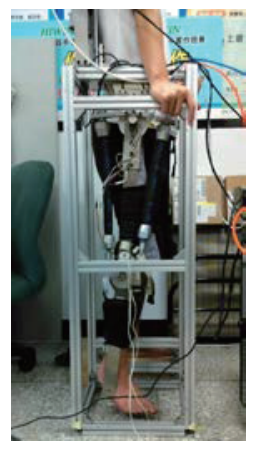

(a)

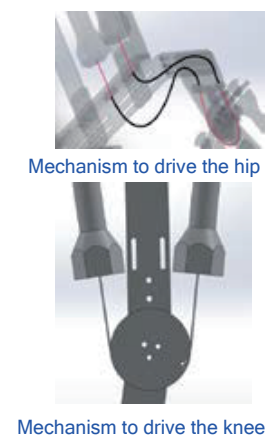

(b)

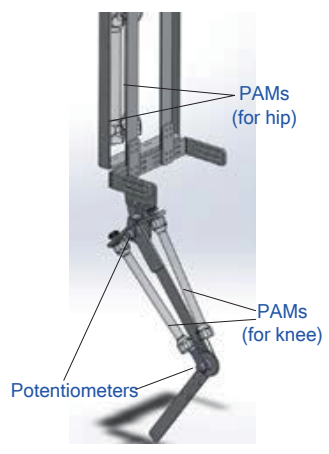

(c)

Fig. 2. (Color online) (a) Photograph of the proposed rehabilitation robot. (b) Driving mechanism for the hip and knee. (c) Robotic mechanism design. 
Fig. 2(b) and each of them is actuated by PAMs. For the knee joint, the first pulley is connected to a steel wire whose two ends are pulled by PAMs (MAS-20-300N-AA-MC-O-ER-BG, made by Festo in Germany) as shown in Fig. 2(c). A similar design is applied to the hip joint, but the hip mechanism is actuated by different PAMs, which are MAS-40-300N-AA-MC-O-ER-BG, because the loading of the hip is larger than that of the knee. Potentiometers with high resolution are used to measure the joint angular positions of the hip and knee of the robot, and then the positioning signals are fed back to the embedded control (NI MyRIO). The real-time control program is developed in Labview language, and position-based trajectory tracking is implemented by propositional-integral-derivative (PID) control. The "teach-and-replay" method is used to generate the rehabilitation trajectory. The training modes are separated into two modes, which are the passive and active modes. The passive mode is usually used in the early stage of rehabilitation, and it should be conducted to help patients track the predefined trajectory to reduce muscle atrophy and improve movement ability. ${ }^{(15)}$ After a specified training period, the advanced stage of rehabilitation is called the active mode, which should be carried out to encourage patients to trigger robot assistance by their own active efforts. ${ }^{(16)}$ To realize the EMG-triggered control to identify the patient's movement intention, in the following section, we will discuss the signal processing of EMG signals, feature extraction, and pattern recognition.

\section{2 cHRi using EMG sensors}

For assisting rehabilitation in the active training mode, cHRi is the key component to manipulate the rehabilitation robotic system. Although EMG enables the assessment of muscle activity, the patient's movement intention cannot be identified from the EMG raw data directly. For example, Fig. 3 shows the EMG signal captured from the patient's lower limb as he raises his leg and then stands in the same cycle time. From the experimental results in Fig. 3, the EMG signal is determined to be very noisy.

Therefore, the processes of filtering, processing, decomposition, and classification for cHRi are necessary. Figure 4 shows the main components of cHRi, which are described as follows.

(1) Filtering and amplifying: The EMG signal is filtered and amplified first. An active band-pass filter circuit is used for this system, and the filter's bandwidth ranges from 7 to $482 \mathrm{~Hz}$. After filtering the noise, the filtered EMG signal is amplified by TL082IC to adjust the suitable voltage range for NI PCI6024E.

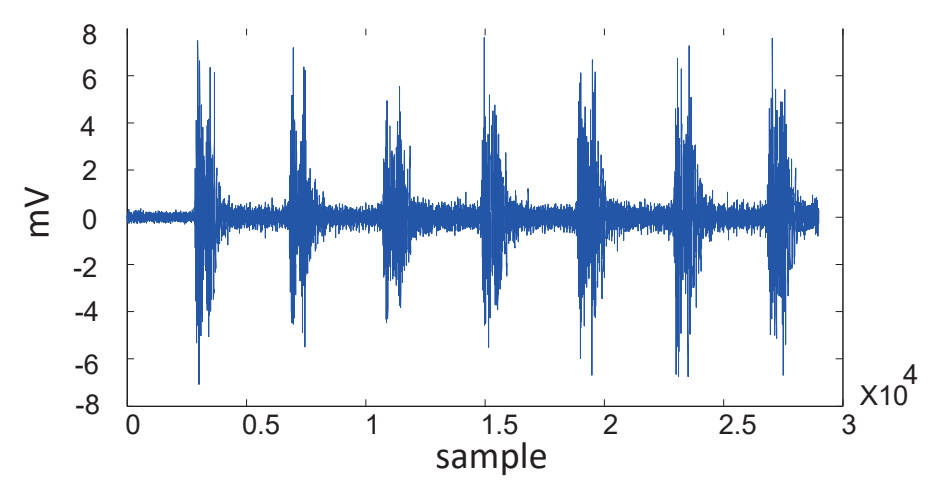

Fig. 3. (Color online) EMG signals captured from the patient's lower limb as he raises his leg and then stands. 


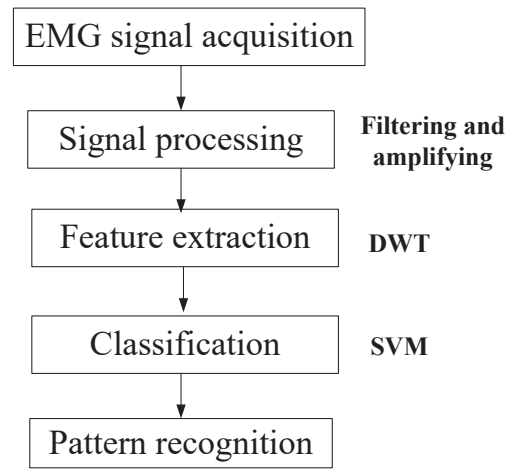

Fig. 4. Proposed method for EMG feature extraction and classification.

(2) Feature extraction: It is difficult to distinguish the important messages from the EMG signal in the time-domain signal, because there is too much noise. Generally, the time-domain signal should be transferred into the frequency-domain power spectrum to analyze the energy components. First, the discrete wavelet transformation (DWT) is used to transfer the EMG signal into the wavelet components. After analyzing the importance of each feature for EMG signals, the best feature for classification can be obtained.

(3) Classification and pattern recognition: The real-time classification of the EMG feature is very important for the EMG-triggered control, because the accuracy and speed of identifying patients' movement intention determine the correctness and real-time performance of the rehabilitation trigger control. Among these classification algorithms, artificial neural networks (ANNs) are widely used in previous studies to improve the recognition accuracy. In recent decades, support vector machines (SVMs) are applied in machine learning for many applications. An SVM is a supervised learning model with associated learning algorithms that analyze the data used for classification and regression analysis. In this study, an SVM is applied to identify the patients' movement intention according to the EMG extracted feature in the wavelet components. In Sect. 3, we will discuss how to obtain the optimal features for classification to identify the patient's movement intention and analyze the classification performance of an SVM.

\section{Feature Extraction by DWT Decomposition for EMG Signals}

Because the time-domain EMG signal is easily affected by noise, it is difficult to distinguish a patient's movement intention directly on the basis of the time-domain signal. If the EMG signals are transferred into the frequency domain, the noise is easily removed; the difference between lifting the patient's leg and the standing state can be easily classified in the frequency domain. Fourier transform (FT) is one of the most popular spectral analysis methods, but it will lose the time information as it is transformed from the time domain into the frequency domain. To improve the disadvantages of the FT, short-time Fourier transform (STFT) was proposed by Dennis Gabor in 1946. It was used as a time-window function at a specific position for signal analysis. ${ }^{(17)}$

However, if a complex of wide-band signals is analyzed, the characteristics of the signals cannot be represented accurately. On one hand, selecting a longer window can provide better frequency-domain analysis, but the time-domain analysis will deteriorate. On the other hand, 
a shorter window will provide excellent time-domain analysis; however, the frequency-domain analysis will become relatively poor. Compared with the STFT, wavelet transformation (WT) has a different window function frame. The WT can change the frame size with respect to the accuracy requirement of the time or frequency analysis. It uses the wavelet function via the time-shift and scaling parameters to induce signal decomposition. Wavelet analysis is like a mathematical camera with a microscope lens. The wavelet function $\psi$ must satisfy the following conditions. ${ }^{(18-20)}$

$$
\int_{-\infty}^{+\infty} \psi(t) d t=0 \text { and } \int_{-\infty}^{+\infty}|\psi(t)|^{2} d t<\infty
$$

A wavelet basis function $\psi_{a, \tau}(t)$ is defined as

$$
\psi_{a, \tau}(t)=\frac{1}{\sqrt{a}} \psi\left(\frac{t-\tau}{a}\right), \mathrm{a}>0
$$

where $1 / \sqrt{a}$ is the normalization factor, $\psi(t)$ is the mother wavelet, and $(1 / \sqrt{a}) \psi(t / a)$ is the dilation of $\psi(t)$ with the factor $a$. The translation factor $\tau$ can make $\psi(t)$ have a time shift. Therefore, the continuous wavelet transform (CWT) can be defined as

$$
C W T(a, \tau)=\frac{1}{\sqrt{a}} \int_{-\infty}^{+\infty} f(t) \psi^{*}\left(\frac{t-\tau}{a}\right) d t
$$

where $f(t)$ is the input signal, CWT $(a, \tau)$ represents continuous wavelet coefficients that are scaling and translation functions, and $\psi^{*}$ is the complex conjugate of $\psi$. For real-time computation, the DWT was proposed to reduce the amount of data and improve the computation speed in the $1980 \mathrm{~s} .{ }^{(18)}$ For the DWT, the wavelet basis function $\psi_{a, \tau}(t)$ should be discretized with respect to the scaling and translation factors. Therefore, the scaling and translation factors of the discrete sampling are given by

$$
a=a_{o}^{j}, \tau=a_{o}^{j} k, j, k \in Z,
$$

where $j$ is the number of scales and $k$ is the number of samples. Then, the wavelet function in the discrete space can be defined as

$$
\psi_{j, k}(t)=\frac{1}{\sqrt{a_{o}^{j}}} \psi\left(\frac{t-a_{o}^{j} k}{a_{o}^{j}}\right), a_{o}>0 .
$$

According to the suggestion in the previous literature, the parameter $a_{o}$ is chosen as 2 and called the dyadic wavelet function, so that Eq. (5) is rewritten as

$$
\psi_{j, k}(t)=\frac{1}{\sqrt{2^{j}}} \psi\left(\frac{t-2^{j} k}{2^{j}}\right)
$$

Then, the DWT is defined as follows according to Eq. (6). 


$$
D W T(j, k)=\frac{1}{\sqrt{2^{j}}} \int_{-\infty}^{+\infty} f(t) \psi^{*}\left(\frac{t-2^{j} k}{2^{j}}\right) d t
$$

The original signal can be decomposed by the DWT into the low-frequency part (approximation) and the high-frequency part (detail). For example, Fig. 5 shows that an original signal is decomposed into two components, namely, $\mathrm{cD}$ and $\mathrm{cA}$, where the original signal is denoted by $\mathrm{S}$, and $\downarrow 2$ denotes the down sampling by a factor of 2 .

The most commonly used set of discrete wavelet transforms was formulated by the Belgian mathematician Ingrid Daubechies in 1988. Mallat proposed that the DWT signal could be decomposed and reconstructed by multiresolution analysis (MRA) in 1989. ${ }^{(21,22)}$ With MRA, the original signal was transferred by discrete wavelet decomposition (DWD) and decomposed into a low-frequency approximation (A1) and a high-frequency detail (D1). ${ }^{(23-25)}$ Then, the first lowfrequency approximation (A1) is decomposed again into a high-frequency detail (D2) and a lowfrequency approximation (A2); similar decompositions can be obtained in accordance with the actual needs of wavelet decomposition layers as shown in Fig. 6. Therefore, we use the DWT for EMG signals to observe the key feature that depends on patient's movement intention. For example, an EMG signal is determined by the decomposition of five layers by DWD as shown in Fig. 7.

The EMG signals are nonstationary signals that vary with the muscle activity. After the decomposition of the DWT, the features of signals cannot be identified clearly. Therefore, seven feature functions are used to analyze the EMG patterns for different situations. The feature functions are listed as follows.

$$
\begin{aligned}
& \text { Root-mean-square value: } \quad R M S=\sqrt{\frac{x_{1}^{2}+x_{2}^{2}+x_{3}^{2}+\ldots x_{n}^{2}}{n}} \\
& \text { Mean absolute value: } \quad M A V=\frac{\left|x_{1}\right|+\left|x_{2}\right|+\left|x_{3}\right|+\ldots\left|x_{n}\right|}{n} \\
& \text { Norm: } \\
& x_{\text {norm }}=\frac{x-\min (x)}{\max (x)-\min (x)}, x \text { is } M A V \text { value } \\
& \text { sum }=\sum_{n=1}^{n} x_{n} \\
& \text { Summation: } \\
& X_{N, \max }=\max _{0 \leq n \leq N}\left(X_{N}(n)\right) \\
& X_{N, \min }=\min _{0 \leq n \leq N}\left(X_{N}(n)\right) \\
& \text { Range: } \\
& X_{N, \text { Range }}=X_{N, \max }-X_{N, \min }
\end{aligned}
$$

The different feature functions according to the different wavelet components can represent different eigenvalues of the EMG signals. Therefore, in the next section, we will discuss the classification analysis using the different feature functions of the different wavelet components to obtain the optimal solution for identifying the movement intention of patients. 


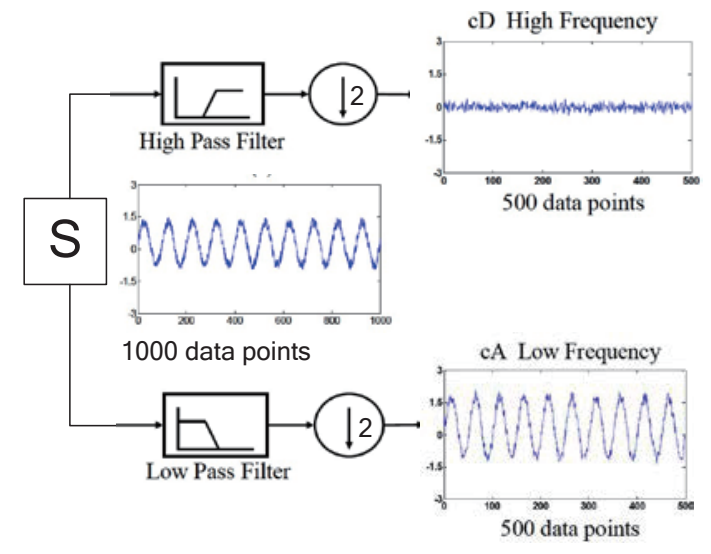

Fig. 5. (Color online) Illustration for the decomposition of DWT.

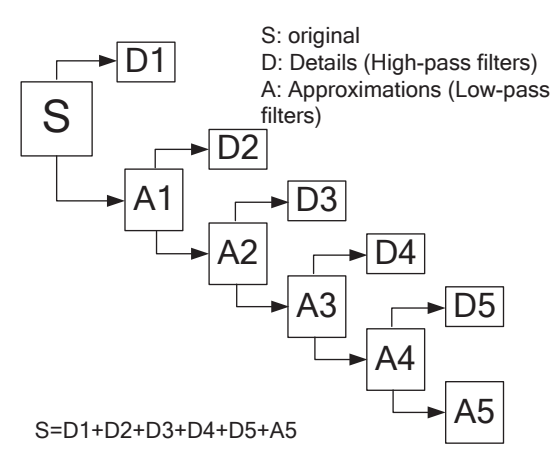

Fig. 6. DWT signal decomposition processing.

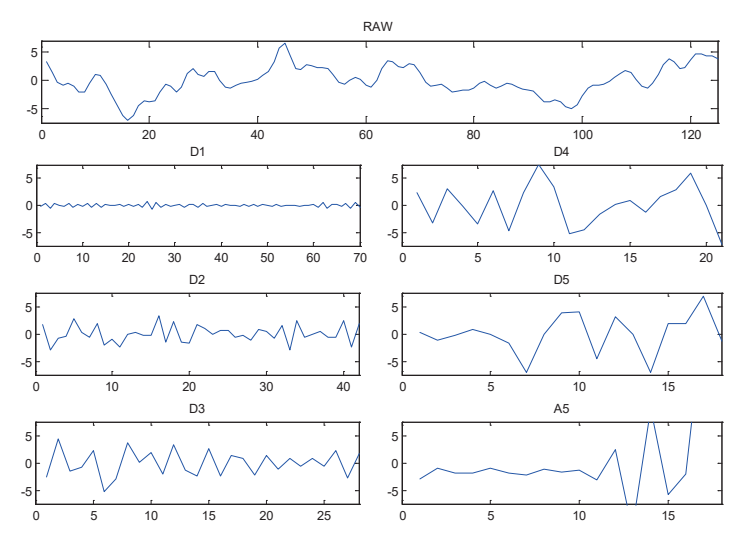

Fig. 7. (Color online) Signal energy in each layer determined by DWD.

\section{Classification of EMG Signals Using SVM}

An SVM constructs a hyperplane or a set of hyperplanes in a multidimensional space, which can be used for classification and data regression for many applications. ${ }^{(26-31)}$ In this case study, good separation depends on the suitable choice of feature functions after DWT decomposition. The EMG raw data are obtained from the specified experiments for eight different persons. Two classification patterns of EMG signals are captured using the DAQ card of NI 6024E. The first pattern is to lift his or her leg and the second pattern is just to stand at rest. The first pattern is used to identify the patient's movement intention; the raw data of EMG signals are recorded when the patient is lifting his or her leg twenty times. The second pattern is established for the pattern of standing at rest.

Case I: To capture the EMG raw data, there are eight different persons lifting their legs or standing at rest twenty times. Therefore, each type of collected EMG signal has 160 group characteristic signal data sets, with a total of 320 group characteristic data sets. Therefore, we can obtain two sets for SVM classification. To find the best choice of SVM classification, the different feature functions in Eqs. (8)-(14) with D3, D4, and D5 in the DWT decomposition are analyzed. 
For this case study, all testing group data are the same as the training group data. By using the different feature pairs for the SVM classifier, the feature pairs of MAV + Max, Norm + RMS, and $N o r m+M A V$ using the D5 wavelet component have the highest recognition rate of $100 \%$, as shown in Table 1.

Case II: For the above classification test in Table 1, all testing group data are the same as the training group data. However, to obtain a robust SVM classifier, the second experiment uses an outside testing method whose testing group data are different from the training group data. Each training group data is not placed in the testing group data. The characteristic signals of each person at different states are divided into a training group set (160 data) and a testing group set (160 data). In the first case study, the SVM classifiers using the feature pairs of MAV + Max, Norm + $R M S$, and Norm + MAV of the D5 wavelet component have the highest recognition rate. Therefore, the second case study focuses on the above three SVM classifiers. Table 2 shows the experimental results for the all-training, half-training, and external testing cases. The results show that the SVM classifiers using the feature pairs of MAV + Max and Norm + MAV have the highest recognition rate of $100 \%$ for the external testing case. Therefore, the proposed real-time trigger control of the PAM rehabilitation robot uses the SVM classifiers of MAV + Max with the D5 wavelet component to identify the movement's intention. Figure 8 shows the real-time experimental result for the PAM rehabilitation robot using the proposed EMG-triggered controller. The first and second figures show the tracking response for the proposed PAM actuated rehabilitation lower-limb robotic system and the third figure shows the captured EMG signal from the patient. The experimental results obtained using the proposed $D W T+S V M$ classifier validate the proposed EMG-triggered controller.

Table 1

Recognition rates for the different feature functions.

\begin{tabular}{lccc}
\hline & D3 (\%) & D4 (\%) & D5 (\%) \\
\hline MAV + Max & 97.18 & 98.43 & 100 \\
MAV + Range & 97.81 & 98.43 & 99.37 \\
Range + Sum & 97.81 & 98.43 & 99.06 \\
Norm + RMS & 97.5 & 98.12 & 100 \\
Norm + MAV & 96.87 & 98.43 & 100 \\
Norm + Sum & 52.19 & 60.6 & 60.31 \\
Norm + Max & 96.87 & 98.75 & 99.06 \\
Norm + Min & 97.5 & 98.43 & 99.06 \\
Norm + Range & 97.81 & 98.43 & 99.06 \\
\hline
\end{tabular}

Table 2

Experimental results for the all-training, half-training, and external testing cases.

\begin{tabular}{lccc}
\hline SVM/D5 & $\begin{array}{c}\text { All training for testing } \\
(\%)\end{array}$ & $\begin{array}{c}\text { Half training for testing } \\
(\%)\end{array}$ & $\begin{array}{c}\text { External testing } \\
(\%)\end{array}$ \\
\hline$M A V+$ Max & 100 & 100 & 100 \\
Norm + RMS & 100 & 99.6 & 98.12 \\
Norm + MAV & 100 & 100 & 100 \\
\hline
\end{tabular}



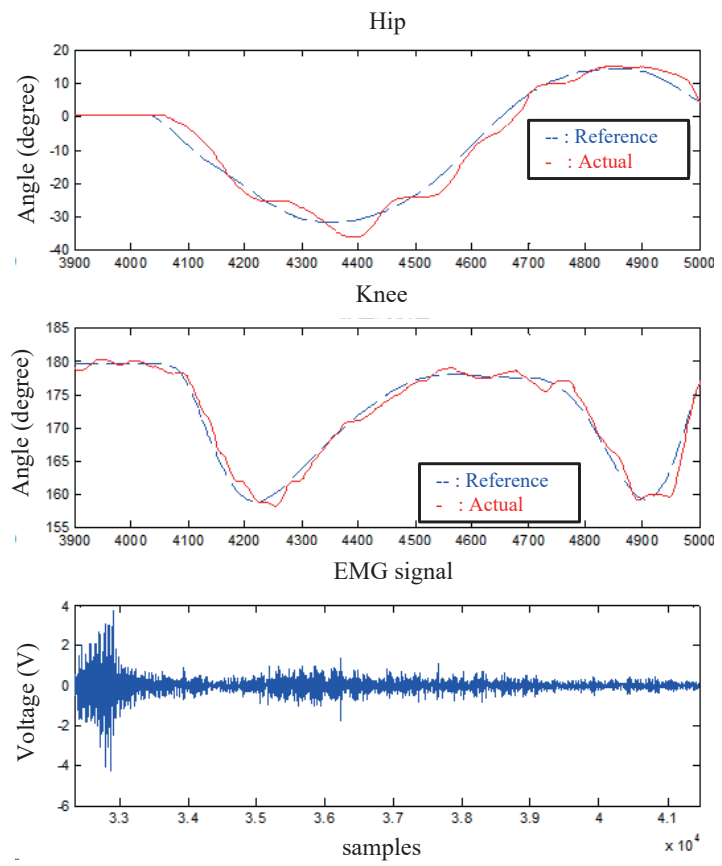

Fig. 8. (Color online) Real-time experimental result obtained using the proposed EMG-triggered control.

\section{Conclusions}

In this paper, we propose a new EMG-triggered controller for the self-designed PAM actuated rehabilitation robotic system. In this study, we focus on how to identify patients' movement intention to realize the proposed EMG-triggered controler. Therefore, we use DWT for EMG signals to observe the key feature that depends on the patient's movement intention. EMG raw signals are decomposed into six components with five-level MRA, which are A5, D1, D2, D3, D4, and D5. To choose the best SVM, the different feature functions with D3, D4, and D5 in the DWT decomposition are analyzed. Using the different feature pairs for an SVM classifier, the feature pairs of MAV + Max, Norm + RMS, and Norm + MAV using the D5 wavelet component have the highest recognition rate of $100 \%$. To test the robustness for external testing cases, the SVM classifiers using the feature pairs of $M A V+$ Max and Norm $+M A V$ have the highest recognition rate of $100 \%$. Therefore, the proposed real-time trigger controller uses the SVM classifiers of MAV+ Max with D5 to identify the movement's intention. The final real-time experimental result for the PAM rehabilitation robot validates the proposed EMG-triggered controller.

\section{Acknowledgments}

The authors would like to thank the Ministry of Science and Technology of the Republic of China, Taiwan for financially/partially supporting this research under Contract Nos. MOST 1042221-E-027-026 and MOST 105-2221-E-027-041. 


\section{References}

1 Z. Zhou, W. Meng, Q. S. Ai, Q. Liu, and X. Wu: Adv. Mech. Eng. (2013) Article ID 574896.

2 I. Diaz, J. J. Gil, and E. Sanchez: J. Robot. 11 (2011).

3 G. Kwakkel, B. J. Kollen, and H. I. Krebs: Neurorehabil. Neural. Repair 22 (2008) 111.

4 S. Mohammed, Y. Amirat, and H. Rifai: Adv. Robot. 26 (2012) 1.

5 W. Huo, S. Mohammed, J. C. Moreno, and Y. Amirat: IEEE Syst. J. 10 (2014) 1068.

6 W. Meng, Q. Liu, Z. Zhou, Q. Ai, B. Sheng, and S. Xie: Mechatronics 31 (2015) 132.

7 B. Tondu: Intell. Mater. Sys. Struct. 23 (2012) 225.

8 N. Costa, M. Berdicek, M. Brown, J. O. Gray, and D. G. Caldwell: Int. J. Autom. Comput. 3 (2006) 271.

9 G. S. Sawicki and D. P. Fessis: J. Neuro-Eng. Rehabil. 6 (2009) 23.

10 Y. Park, B. Chen, D. Young, L. Stirling, R. Wood, E. Goldfield, and R. Nagpal: Proc. 2011 Int. Conf. Robots and Systems (IROS 2011).

11 C. M. Teng, Z. Y. Wong, W. Y.The, and Y. Z. Chong: Proc. 2012 Int. Conf. Biomedical Engineering (ICoBE 2012).

12 S. Hussain, S. Q. Xie, and P. K. Jamwal: IEEE Trans. Cybern. 43 (2013) 1025.

13 M. Arzuwan, M. Dzahir, and S. I. Yamamoto: Robotics 3 (2014) 120.

14 C. J. Lin, C. R. Lin, S. K. Yu, and C. T. Chen: Mechatronics 28 (2015) 35.

15 S. Pittaccio and S. Viscuso: J. Mater. Eng. Perform. 20 (2011) 666.

16 W. Meng, Q. Liu, Z. Zhou, and Q. Ai: Ind. Robot 41 (2014) 465.

17 D. Gabor: J. Inst. Electr. Eng. Part III: Radio Comm. Eng. 93 (1946) 429.

18 I. Daubechies, A. Grossmann, and Y. Meyer: J. Math. Phys. 27 (1986) 1271.

19 C. S. Chen, C. L. Huang, and C. W. Yeh: Smart Sci. (2016) 117.

20 L. C. Chen, C. Y. Chang, W. C. Lee, and C. C. Ma: Smart Sci. (2016) 80.

21 S. Mallat: IEEE. Trans. Pattern Anal. Mach. Intell. 11 (1989) 674.

22 S. Mallat and S. Zhong: IEEE Trans. Pattern Anal. Mach. Intell. 14 (1992) 710.

23 W. Wang, H. Tan, Y. Pang, Z. Li, P. Ran, and J. Wu: J. Sens. (2016) Article ID 2646205.

24 G. Han, J. Wang, and X. Cai: Sensors 16 (2016) 456.

25 N. R. Kidwai, E. Khan, and M. Reisslein: IEEE Sensors J. (2016) 2575.

26 C. Cortes and V. Vapnik: Mach. Learn. 20 (1995) 273.

27 Y. Sakumura, Y. Koyama, H. Tokutake, T. Hida, K. Sato, T. Itoh, T. Akamatsu, and W. Shin: Sensors 17 (2017) 287.

28 P. Huang, Y. Jin, D. Hou, J. Yu, D. Tu, Y. Cao, and G. Zhang: Sensors 17 (2017) 581.

29 F. Zhu and J. Wei: J. Nanoelectron. Optoelectron. 12 (2017) 452.

30 Z. Feng and J. Fu, D. Du, F. Li, S. Sun: Int. J. Distrib. Sens. Netw. 13 (2017) 1.

31 T. Liu and T. Wu: Smart Sci. (2017) 150.

\section{About the Authors}

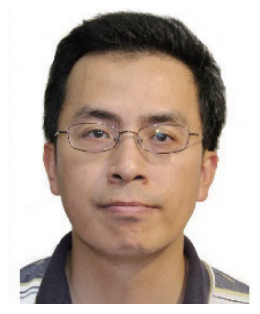

Chih-Jer Lin received his B.S., M.S., and Ph.D. degrees from the National Cheng Kung University, Tainan, Taiwan, in 1992, 1994, and 1998, respectively, all in mechanical engineering. He is currently a professor of the Graduate Institute of Automation and Technology, National Taipei University of Technology, Taipei, Taiwan. His current research interests include mechatronics, vibration control, motion control, system identification, sliding-mode control, robotics, and evolutionary algorithms. Professor Lin has been a guest editor of Applied Sciences and a member of the editorial board of the Journal of Chinese Society of Mechanical Engineers. 


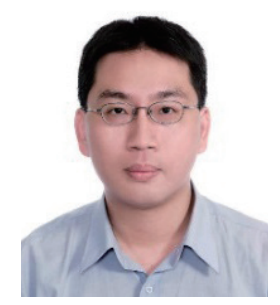

Ho-Chiao Chuang received his B.S. in mechanical engineering at Da-Yeh University, Taiwan, in 2000 and M.S. in the Department of Power Mechanical Engineering at National Tsing-Hua University, Taiwan, in 2002. He joined Professor Victor Bright's MEMS group in 2004 and earned his Ph.D. in 2008 from the Department of Mechanical Engineering at the University of Colorado at Boulder, USA. He joined the Department of Mechanical Engineering, National Taipei University of Technology, Taiwan, in 2009. His current research interests include the fabrication of through-silicon vias (TSVs) for 3D ICs, the ultrahigh aspect ratio of the TSV by using the supercritical $\mathrm{CO}_{2}$ electroplating process, the development of new biomedical instruments especially in the aspect of counteraction treatment couch for respirationinduced tumor motion, and the development of novel energy-saving technology for different application fields.

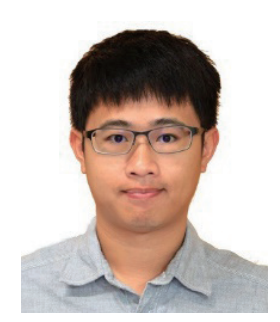

Chao-Wei Hsu received his B.S. and M.S. degrees from the National University of Technology, Taiwan, in 2013 and 2015, respectively. From 2015 to 2016, he fulfilled his mandatory military service. He is currently an engineer in WINSTAR Display. His research interests are in mechatronics, soft computing, and sensors.

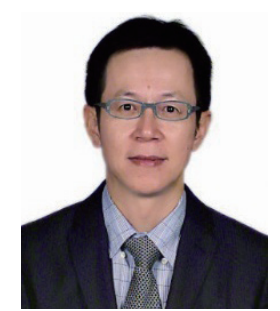

Chin-Sheng Chen received his Ph.D. degree in mechanical engineering from the National Chiao Tung University, Hsinchu, Taiwan, in 1999. He was a Researcher with Sintec Technology Co., Ltd. from 1999 to 2000 and an R\&D manager with TECO Electric and Machinery Co., Ltd. from 2000 to 2002. In 2002, he joined the Graduate Institute of Automation Technology, National Taipei University of Technology, Taiwan, as an assistant professor. Presently, he is a professor and a director of the Graduate Institute of Automation Technology at the National Taipei University of Technology. His research interests include intelligent motion control, mechatronics, and machine vision. He received the Outstanding Research Award from the College of Mechanical and Electrical Engineering, National Taipei University of Technology in 2013 and 2014. Professor Chen has published over 150 journal and conference papers and book chapters. 